\title{
Epidemiology of deep venous thrombosis during pregnancy and puerperium in Sudanese women
}

\author{
Asha A Gader' \\ Abed Elrahium D Haggaz ${ }^{2}$ \\ Ishag Adam' \\ 'Faculty of Medicine, University \\ of Khartoum, Sudan; \\ ${ }^{2}$ Department of Obstetrics \\ and Gynecology, Faculty of Medicine \\ University of Elfasher, Sudan
}

\begin{abstract}
Background: Deep venous thrombosis (DVT) and venous thromboembolism (VTE) is a major health problem with high mortality worldwide. Patients at risk must be identified and given appropriate prophylaxis in order to decrease the mortality.

Objective: To investigate the prevalence of DVT in pregnancy and the puerperium and to identify risk factors for DVT.

Setting: Khartoum and Khartoum North Teaching hospitals, Sudan.

Design: Case-control study.

Results: During the study period (April 2007-March 2008), 65 patients presented with DVT as confirmed by Doppler ultrasound. A total of 14,490 deliveries occurred during the study period. The rate was 448 DVT per 100,000 births/year. Only four of these 65 patients were pregnant and the rest presented at postpartum. DVT occurred in the left lower extremity in $51(78.4 \%)$, in the right in $13(20.0 \%)$, and in one (1.5\%) woman in both legs. In univarite and multivariate analyses, family history of DVT, primigravidae and cesarean section deliveries showed an higher risk of DVT.

Conclusions: The study showed a high prevalence of DVT, most of these events occurred in the postpartum period. Primiparae, family history of DVT and cesarean section deliveries were important risk factors and these groups are candidate for prophylaxis measures against DVT.
\end{abstract}

Keywords: DVT, pregnancy, puerperium, risk, Sudan

\section{Introduction}

Deep venous thrombosis (DVT) and venous thromboembolism (VTE) is a major health problem with high mortality worldwide. Multiple changes occur in the coagulation system during pregnancy. Consequently pregnant women are more susceptible to VTE. Therefore, the risk of VTE is much higher in a pregnant woman than in a nonpregnant woman of similar age. ${ }^{1}$ Sometimes death due to VTE is so rapid that it leaves insufficient time for intervention. Patients at risk must be identified and given appropriate prophylaxis to reduce VTE-related mortality. The failure to reduce this rate may be a result of uncertainty regarding risk factors for VTE and the associated difficulty in recognizing individuals at risk. Reported risk factors vary widely as do the genetic, environmental and behavioral factors. ${ }^{2,3}$

The current study was conducted to investigate the epidemiology of DVT in pregnancy and puerperium and to identify its risk factors, which include the ABO blood group system among Sudanese women. This will help health planners and caregivers to identify and hence prevent DVT in those women at risk.

\section{Materials and methods}

A case-control study was conducted at Khartoum, Khartoum North hospitals, Sudan during a one-year period of April 1st, 2007-March 13, 2008. 
The study aimed to investigate prevalence, timing, and the risk factors for DVT in pregnancy and puerperium in a population of women in central Sudan. Consecutive patients presented with symptoms suggestive of DVT to the obstetrics and gynecology ward were approached for participation in the study. DVT was confirmed or excluded objectively by Doppler ultrasound. For each case, two consecutive controls were chosen from the population of patients presenting to the same unit without symptoms of VTE. Cases and controls completed a questionnaire of personal data and clinical history. Questions regarding known risk factors for VTE such as: age parity, preeclampsia, mode of delivery, history of using oral contraceptive pills (OCP), past history and family history of DVT were included. Blood groups and hemoglobin were investigated.

\section{Statistic analysis}

Data were entered into a computer database and SPSS software (SPSS Inc., Chicago, IL, USA) and double checked before analysis.

Univarite and multivariate analyses were performed with odd ratio and $95 \%$ CI calculated, DVT was a dependent variable and maternal age, parity, mode of delivery, preeclampsia, past history and family history of DVT and blood group were independent variables. A P-value $<0.05$ was considered significant.

\section{Ethics}

The study received ethical clearance from the Research Board at the Faculty of Medicine, University of Khartoum.

\section{Results}

Sixty-five patients presented with symptoms and signs of VTE during the study and this was confirmed by
Doppler ultrasound. A total of 14,490 deliveries occurred at these hospitals during the study period. There were 448 DVT per 100,000 births/year.

Only four of these 65 women were pregnant and the rest were postpartum. Among those pregnant women, three experienced deep vein thrombosis in the third trimester, one in the second. DVT occurred in the left lower extremity in $51(78.4 \%)$, in the right in $13(20.0 \%)$, and in one $(1.5 \%)$ woman in both legs.

In univarite and multivariate analyses, family history of DVT, primigravidae and cesarean section deliveries showed an higher risk of DVT. Those with past history of DVT were found at higher risk using univarite analyses only (Table 1).

\section{Discussion}

The main results of the current study are: the high incidence of DVT; the susceptibility of women to DVT after the delivery or late during pregnancy; the left side was most often affected. Primigravidae women, those women who have past history of DVT and cesarean section deliveries, were at higher risk of DVT. Preeclampsia, body mass index, and blood groups were not predictors for DVT. This was a hospital-based study and the vast majority of Sudanese women deliver their children in their home (Adam pers comm). This could explain the relatively high prevalence of DVT in our study. Our results were in agreement with previous reports as there were approximately twice and five times as many postpartum as antepartum DVT events. ${ }^{4,5}$ In contrast, James and colleagues reported more pregnant women suffer DVT in early pregnancy than in the puerperium. ${ }^{6}$ In a meta-analysis, it was reported that about $82 \%$ of DVT occurred in the left lower extremity. Though the exact cause is not known, anatomic reasons have been postulated. ${ }^{6,7}$ In our traditions, the first delivery is the

Table I Risk factors for DVT in Sudanese women using unviarite and multivariate analyses

\begin{tabular}{|c|c|c|c|c|c|c|}
\hline \multirow[b]{2}{*}{ Variable } & \multicolumn{3}{|c|}{ Univarite analyses } & \multicolumn{3}{|c|}{ Multivariate analyses } \\
\hline & OR & $95 \% \mathrm{Cl}$ & $P$ & OR & $95 \% \mathrm{Cl}$ & $P$ \\
\hline Age, years & 0.8 & $0.8-0.9$ & 0.009 & 1.06 & $1.0-1.2$ & 0.1 \\
\hline Primigravidae & 2.2 & $1.2-4.3$ & 0.01 & 2.7 & $1.2-4.9$ & 0.01 \\
\hline Family history of DVT & 7.4 & $2.3-24.2$ & 0.001 & 5.9 & $1.68-30.5$ & 0.005 \\
\hline Past history of DVT & 6.3 & $1.6-24.3$ & 0.008 & 4.7 & $0.9-25.0$ & 0.06 \\
\hline Cesarean section delivery & 2.0 & $1.0-3.8$ & 0.02 & 2.2 & I.I-4.4 & 0.02 \\
\hline Body mass index & 1.0 & $0.9-1.0$ & 0.4 & 1.0 & $0.9-1.0$ & 0.4 \\
\hline \multicolumn{7}{|l|}{ Blood group } \\
\hline$A$ vs none $A$ & 1.8 & $0.9-1.8$ & 0.08 & 2.0 & $0.8-4.9$ & 0.1 \\
\hline O vs none $\mathrm{O}$ & 0.7 & $0.4-1.3$ & 0.3 & 0.4 & $0.08-2.1$ & 0.2 \\
\hline Preeclampsia & 0.9 & $0.4-2.2$ & 0.9 & 1.0 & $0.4-2.5$ & 0.8 \\
\hline
\end{tabular}

Abbreviation: DVT, deep venous thrombosis. 
most popular event, primiparae women - with relatively no or little experience of childbirth - would be under the mercy of these traditions. One of these traditions is the restriction of movement, which primigravidae women have to obey and this could explain their susceptibility to DVT in this study. Bed rest or immobility, pelvic or leg trauma have previously been reported to be risk factors for DVT. ${ }^{2}$

Although epidemiologic studies have demonstrated many risks a deficit of blood group $\mathrm{O}$ among patients with DVT, few studies have evaluated the importance of blood group in these high-risk patients. Recently non-O blood type was independently associated with risk of VTE. ${ }^{3}$ Larsen and colleagues reported the susceptibility of women with blood group B and AB to DVT than the other with A or O blood group. ${ }^{7}$ On the other hand, Carter and colleagues failed to show an association between DVT and blood group in high risk patients. ${ }^{9}$ Yet blood group A, multiple pregnancy, caesarean section, cardiac disease, preterm delivery, high body mass index and elder women ( $>35$ years) were found to increase incidence of VTE. ${ }^{4}$ The association of blood group with DVT could explore the genetic predisposition (Factor V Leiden) of these groups to DVT. Although, we failed to observe an association between blood group and DVT, but those women who had family history of DVT were at higher risk of DVT.

In the current study, cesarean delivery was a predictor for DVT $(\mathrm{OR}=2.21 ; 95 \% \mathrm{CI}=1-4.40, \mathrm{P}=0.02$. Cesarean section is a known risk factor for DVT. ${ }^{2}$ However, one of the studies failed to observe the risk of DVT among pregnant women undergoing cesarean section. ${ }^{10}$

Smoking is not unusual among Sudanese women, hence it was not investigated in the current study. Due to limited resources, thrombophilias were not screened; it would be of great value to be included in the future research especially family history of DVT was found to be significant in this study. This was a relatively small sized hospital-based study, with an inherent bias. Therefore these women might not be the true picture of the community. During the study period there was no case of VTE. There incidence of VTE was reported to be lower than the incidence of DVT. ${ }^{5}$ Furthermore, some women with VTE might have presented and treated elsewhere (medical department) or even died without being diagnosed as VTE.

In summary, these groups of women, such as primiparae with a family history of DVT, and women who delivered by cesarean route and had been found at higher risk for DVT should be given prophylaxis measures against DVT.

\section{Disclosure}

The authors report no conflicts of interest in this work.

\section{References}

1. Clark P, Brennand J, Conkie JA, McCall F, Greer IA, Walker ID Activated protein $\mathrm{C}$ sensitivity, protein $\mathrm{C}$, and protein $\mathrm{S}$ and coagulation in normal pregnancy. Thromb Haemost. 1998;79:1166-1170.

2. Colman-Brochu S. Deep vein thrombosis in pregnancy. MCN Am J Matern Child Nurs. 2004;29:186-192.

3. Ohira T, Cushman M, Tsai MY, et al. Folsom ARABO blood group, other risk factors and incidence of venous thromboembolism: the Longitudinal Investigation of Thromboembolism Etiology (LITE). J Thromb Haemost. 2007;5:1455-1456.

4. Simpson EL, Lawrenson RA, Nightingale AL, Farmer RD. Venous thromboembolism in pregnancy and the puerperium: incidence and additional risk factors from a London perinatal database. BJOG. 2001;108:56-60.

5. Heit JA, Kobbervig CE, James AH, Petterson TM, Bailey KR, Melton LJ $3 \mathrm{rd}$. Trends in the incidence of venous thromboembolism during pregnancy or postpartum: a 30-year population-based study Ann Intern Med. 2005;143:697-706.

6. James AH, Tapson VF, Goldhaber SZ. Thrombosis during pregnancy and the postpartum period. Am J Obstet Gynecol. 2005;193:216-219.

7. Ginsberg JS, Brill-Edwards P, Burrows RF, et al. Venous thrombosis during pregnancy: leg and trimester of presentation. Thromb Haemost. 1992;67:519-520.

8. Larsen TB, Johnsen SP, Gislum M, Møller CA, Larsen H, Sørensen HT. $\mathrm{ABO}$ blood groups and risk of venous thromboembolism during pregnancy and the puerperium. A population-based, nested case-control study. J Thromb Haemost. 2005;3:300-304.

9. Carter YM, Caps MT, Meissner MH. Deep venous thrombosis and $\mathrm{ABO}$ blood group are unrelated in trauma patients $J$ Trauma. 2002;52:112-116.

10. Jacobsen AF, Drolsum A, Klow NE, Dahl GF, Qvigstad E, Sandset PM. Deep vein thrombosis after elective cesarean section. Thromb Res 2004;113:283-288. 
